\title{
Clusters in neutron-rich light nuclei
}

\author{
D. Jelavić Malenica ${ }^{1}$, M. Milin ${ }^{2}$, A. Di Pietro ${ }^{3}$, P. Figuera $^{3}$, \\ M. Lattuada ${ }^{3}$, D. Miljanić ${ }^{1}$, A. Musumarra ${ }^{3}$, M.G. Pellegriti ${ }^{3}$, \\ L. Prepolec ${ }^{1}$, V. Scuderi ${ }^{3}$, N. Skukan ${ }^{1}$, N. Soić ${ }^{1}$, D. Torresi ${ }^{3}$ and \\ M. Uroić ${ }^{1}$ \\ 1 Ruder Bošković Institute, Zagreb, Croatia \\ 2 Faculty of Science, University of Zagreb, Zagreb, Croatia \\ ${ }^{3}$ INFN - Laboratori Nazionali del Sud and Sezione di Catania, Catania, \\ Italy
}

\begin{abstract}
Due to their high selectivity, transfer and sequential decay reactions are powerful tools for studies of both single particle (nucleon) and cluster states in light nuclei. Their use is particularly simple for investigations of $\alpha$-particle clustering (because $\alpha$-particle has $\mathrm{J}^{\pi}=0^{+}$, which simplifies spin and parity assignments to observed cluster states), but they are also easily applicable to other types of clustering. Recent results on clustering in neutron-rich isotopes of beryllium, boron and carbon obtained measuring the ${ }^{10} \mathrm{~B}+{ }^{10} \mathrm{~B}$ reactions (at 50 and $72 \mathrm{MeV}$ ) are presented. The highly efficient and segmented detector systems used, built from 4 Double Sided Silicon Strip Detectors (DSSSD) allowed detection of double and multiple coincidences and, in that way, studies of states populated in transfer reactions, as well as their sequential decay.
\end{abstract}

Clustering was recognized [1] as an essential ingredient in description of light nuclei already in the early days of nuclear physics, even before the actual formulation of nuclear shell model. A systematic picture of the phenomenon for the $\alpha$-conjugate nuclei (with $N=Z$ ) emerged in the late sixties, 
resulting in insights like threshold rule (depicted in Ikeda diagram, [2]) or parity splitting of rotational bands of asymmetric cluster states [3]. Next decades of both theoretical and experimental research established the picture, though number of questions remained open (see e.g. $[4,5]$ ). In the new millennium the appearance of clustering was theoretically obtained even within ab initio calculations - e.g. the classical example of clustering, description of the ${ }^{8} \mathrm{Be}$ ground state as two touching $\alpha$-particles, was nicely reproduced within the Green function Monte Carlo ab initio calculations [6].

Isotopes of light nuclei having additional neutrons (compared to the $N=Z$ ones) show a different type of clustering, with extra neutrons acting as valence particles in molecule-like structures. The best example of such clustering is found among states of beryllium isotopes ${ }^{9-12} \mathrm{Be}[4,5]$ - neutrons fill different "molecular orbits" around the existing two-centre $(\alpha-$ $\alpha)$ structure and corresponding states have been identified [7]. The simplest molecular orbits are $\sigma$-orbits which possess an increased density along the axis connecting the two cluster centers, and $\pi$-orbits in which neutrons occupy a ring orbit perpendicular to that axis and centered on it. Delocalization of neutrons in the two-centered orbits reduces their kinetic energies giving thus a strong contribution to the stability of the structure. The Pauli repulsion effect of neutrons in $\sigma$-orbits can furthermore push away two cores to larger distances; such states are predicted [4] to be among the strongest deformed nuclear configurations. For this reason $\sigma$-orbits play an important role in stabilization of all linear chain structures. States with neutrons in $\pi$ - and $\sigma$-orbits are successfully theoretically modeled for ${ }^{10} \mathrm{Be}$ within the framework of antisymmetrized molecular dynamics.

This simplified picture is complicated with additional configurations - e.g. Ito and Itagaki $[8,9]$ distinguish between "ionic", "covalent" and "atomic" states in ${ }^{10} \mathrm{Be}$ and ${ }^{12} \mathrm{Be}$, and further types of states in ${ }^{10} \mathrm{Be}$ are proposed in refs. $[10,11]$. Recently the molecular states in ${ }^{10} \mathrm{Be}$ are also reproduced within the framework of energy density functionals, without any presumption of clustering [12].

Experimental search for nuclear molecules is ongoing for more than 20 years (e.g. [13]), with associate states finally clearly established in ${ }^{10} \mathrm{Be}$ $[14,15]$ as a rotational band starting with the $0_{2}^{+}$state at $E_{x}=6.18 \mathrm{MeV}$. The band is confirmed and extended in subsequent experiments $[16,17]$. Molecular states are predicted to exist in a number of other nuclei (see ref [4] for a recent overview), but all of them have in common the fact that the valence nucleons are neutrons. Due to the Coulomb repulsion, protons as valence particles are expected to make the molecules less stable, especially 
while filling the $\sigma$-orbits. The effect is not expected to prohibit the existence of proton molecular states, although in some cases it may make them rather broad and thus hard to detect. The well-known example is the $1 / 2^{+}$state in ${ }^{9} \mathrm{Be}\left(E_{x}=1.684 \mathrm{MeV}\right)$, for which the ${ }^{9} \mathrm{~B}$ analog is still not clearly identified.

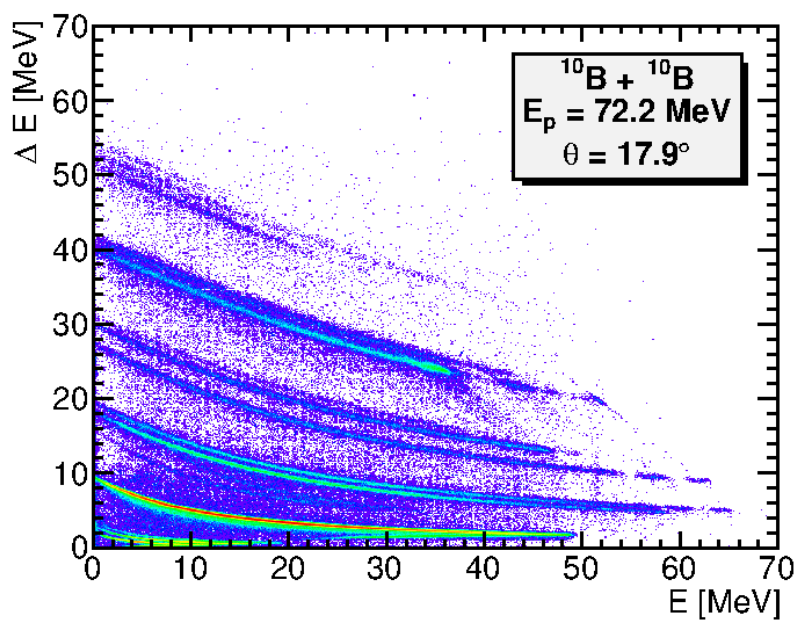

Figure 1: Particle identification spectrum for one of the quadrants of a forward angle telescope. Loci corresponding to different hydrogen, helium, lithium, beryllium, boron and carbon isotopes can be clearly seen.

In order to further study the structure of nuclei in the $A=10$ mass region, the ${ }^{10} \mathrm{~B}+{ }^{10} \mathrm{~B}$ reactions were measured at beam energies of 50 and $72.2 \mathrm{MeV}$. The experiment was performed at INFN-LNS, using the SMP Tandem accelerator and targets enriched in ${ }^{10} \mathrm{~B}$ up to $99.8 \%$. Reaction products were detected with a highly segmented detector setup covering a large solid angle and allowing for the detection of single events as well as twoand three-particle coincidences. The selectivity of the ${ }^{10} \mathrm{~B}+{ }^{10} \mathrm{~B}$ reactions in populating different states of neighbouring nuclei was studied, together with a sequential decay of states in question.

The complex structure of the low-lying states of the ${ }^{10} \mathrm{~B}$ nucleus, which can be described as a mixture of shell model and cluster configurations of the type ${ }^{6} \mathrm{Li}_{g s}+\alpha$ or ${ }^{6} \mathrm{Li}\left(0_{2}^{+}, 1\right)+\alpha$, together with a high spin of the ground state $\mathrm{J}^{\pi}=3^{+}$, enables population of a range of different high-spin states at high excitation energies. Among excited states of ${ }^{10} \mathrm{~B}$ one should also 
look for molecular states analog to the ${ }^{10} \mathrm{Be}$ ones, but with the neutron and proton pair as valence particles, however due to rather different structure of these states with respect to the ${ }^{10} \mathrm{~B}$ ground state, they are not expected to be populated strongly in this experiment.

Detector setup consisted of four $\Delta \mathrm{E}-\mathrm{E}$ silicon telescopes, each composed of thin $\Delta \mathrm{E}$ detector $(57-67 \mu \mathrm{m})$, divided into 4 quadrants and thick E DSSSD detector (500 or $1000 \mu \mathrm{m}$ ), divided into 16 strips in both front and back sides.

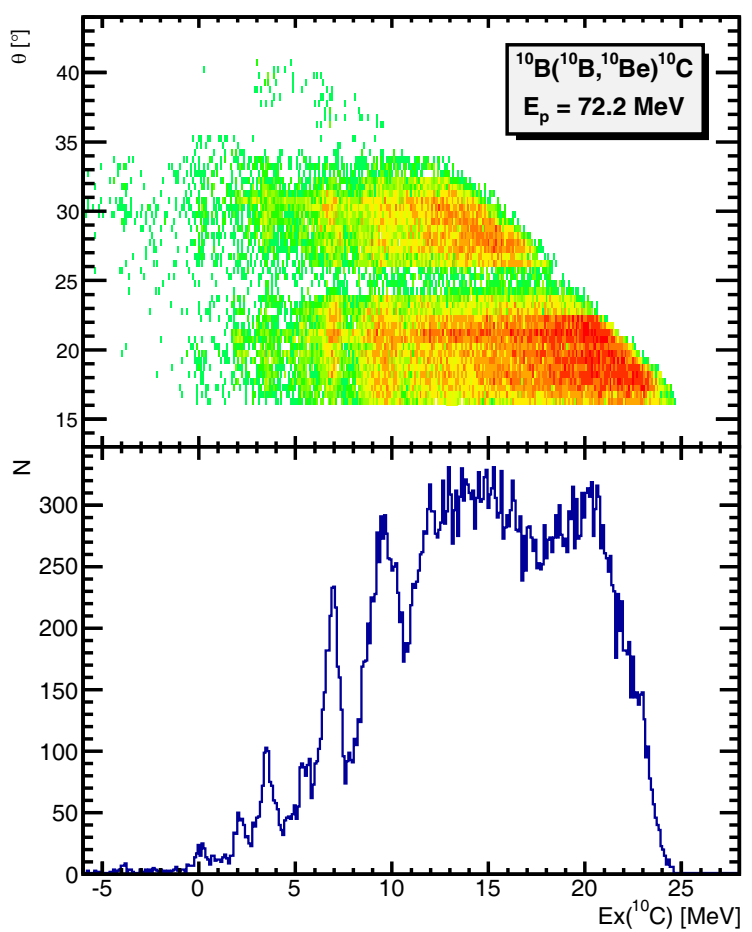

Figure 2: Inclusive spectra of the ${ }^{10} \mathrm{C}$ excitation energy (below) and its angular dependence (above).

Due to the large size of all detectors $\left(50 \times 50 \mathrm{~mm}^{2}\right)$ and their fine segmentation, telescopes covered rather large solid angle with good angular resolution. This same segmentation makes the analysis of obtained experimental data rather demanding - complications associated with dead layers [18] or interstrip gaps $[19,20]$ are only part of the problem, the very basic one being a calibration of a large number of apparently independent detector channels. 
Several novel calibration improvements described in detail in ref. [21] were used in the analysis, as well as a new set of tools that allows an easy and accurate calibration of DSSSD detectors [22].

On both beam energies (50 and $72 \mathrm{MeV}$ ) the number of detected $\alpha$ particles was remarkably higher than number of any other detected nuclei, as can be seen in an example of the particle identification spectra in Fig. 1. This effect was even more pronounced in coincidence measurements, where even triple $\alpha$-particle coincidences were detected with rather large statistics.

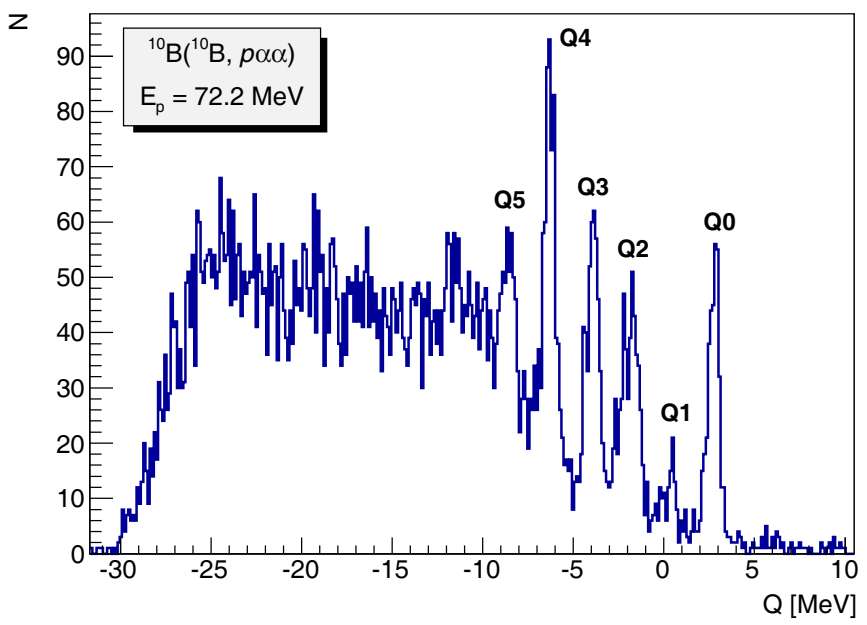

Figure 3: $Q$-spectrum reconstructed from the triple coincidences $\alpha+\alpha+p$ (see text for details).

Applicability of the used detector set-up (with highly segmented strip detectors) for studies of multiple coincidences is nicely illustrated through the spectrum given in Fig. 3. It shows a $Q$-spectrum reconstructed from the triple coincidences $\alpha+\alpha+p$ with several additional assumptions: (i) two $\alpha$ particles have relative energy corresponding to the ground state of ${ }^{8} \mathrm{Be}$; (ii) the reconstructed ${ }^{8} \mathrm{Be}$ and proton have relative motion corresponding to the ground state of ${ }^{9} \mathrm{~B}$ (no other ${ }^{9} \mathrm{~B}$ state is clearly seen in the data). With such cuts the spectrum actually shows the ${ }^{11} \mathrm{~B}$ states populated in the reaction ${ }^{10} \mathrm{~B}\left({ }^{10} \mathrm{~B},{ }^{9} \mathrm{~B}_{\text {g.s. }}\right){ }^{11} \mathrm{~B}$, which was of course never measured before due to the fact that ${ }^{9} \mathrm{~B}$ is particle unbound. The selectively excited ${ }^{11} \mathrm{~B}$ states shown in Fig. 3 are the mirror states of the ${ }^{11} \mathrm{C}$ ones seen in the ${ }^{10} \mathrm{~B}\left({ }^{10} \mathrm{~B},{ }^{9} \mathrm{Be}\right){ }^{11} \mathrm{C}$ 
reaction [22]. In these spectra several states not yet reported in the nucleon transfer reactions on ${ }^{10} \mathrm{~B}$ are seen at higher excitation energies.

A number of other double and triple coincidence spectra is obtained in the experiment, yielding new results for ${ }^{10} \mathrm{~B},{ }^{11} \mathrm{~B},{ }^{11} \mathrm{C},{ }^{12} \mathrm{C},{ }^{13} \mathrm{C}$ and ${ }^{14} \mathrm{~N}$. As expected, preferably populated are the states at high excitation energies and spins (details will be given in subsequent publications).

Several inclusive spectra from the experiment also show interesting results - one of them is given in Fig. 2 for the ${ }^{10} \mathrm{~B}\left({ }^{10} \mathrm{~B},{ }^{10} \mathrm{Be}\right){ }^{10} \mathrm{C}$ reaction. Several peaks correspond to the known ${ }^{10} \mathrm{C}$ (and ${ }^{10} \mathrm{Be}$ ) states, but the peak seen at $E_{x}=9.45 \mathrm{MeV}$ does not. The energy of $9.45 \mathrm{MeV}$ can be obtained combining the 3.35 excitation in ${ }^{10} \mathrm{C}$ with the $\approx 6 \mathrm{MeV}$ excitation in ${ }^{10} \mathrm{Be}$, but since there is no significant peak at $\approx 6 \mathrm{MeV}$ in the spectrum in Fig. 2, this contribution is probably weak, and the peak mainly corresponds to a ${ }^{10} \mathrm{C}$ state at $E_{x}=9.45 \mathrm{MeV}$. The state is not yet reported in the TUNL compilation [23] or later experiments related to ${ }^{10} \mathrm{C}$ (see e.g. [24]); it is most likely the ${ }^{10} \mathrm{C}$ isospin analog of the $2^{+}$state in ${ }^{10} \mathrm{Be}$ at $E_{x}=9.56 \mathrm{MeV}$.

Table 1: Analog states in ${ }^{10} \mathrm{Be},{ }^{10} \mathrm{~B}$ and ${ }^{10} \mathrm{C}$. The numbers in brackets give the excitation energy of the states relative to the lowest $T=1$ state in ${ }^{10} \mathrm{~B}$ (at $\left.1.74 \mathrm{MeV}\right)$.

\begin{tabular}{|c|c|c|c|}
\hline \hline$J^{\pi}$ & $E_{x}\left({ }^{10} \mathrm{Be}\right)$ & $E_{x}\left({ }^{10} \mathrm{~B}\right)$ & $E_{x}\left({ }^{10} \mathrm{C}\right)$ \\
\hline & $(\mathrm{MeV})$ & $(\mathrm{MeV})$ & $(\mathrm{MeV})$ \\
\hline \hline $0^{+}$ & 0.00 & $1.74(0.00)$ & 0.00 \\
$2^{+}$ & 3.37 & $5.16(3.42)$ & 3.35 \\
$2^{+}$ & 5.96 & $7.46(5.81)$ & \\
$1^{-}$ & 5.96 & $7.43(5.69)$ & \\
$0^{+}$ & 6.18 & $7.56(5.82)$ & 5.38 \\
$2^{-}$ & 6.26 & $7.48(5.74)$ & \\
$3^{-}$ & 7.37 & $8.89(7.15)$ & \\
$2^{+}$ & 7.54 & $8.89(7.15)$ & 6.58 \\
$2^{+}$ & 9.56 & & 9.45 \\
\hline
\end{tabular}

The list of the known analog states in $A=10$ nuclei is given in the table 1. One can see that the states with compact geometry (like e.g. ground state) have rather similar excitation energies in all three isotopes, while the well deformed (clusterized) states (like e.g. $0_{2}^{+}$) have energies which are very different. The ${ }^{10} \mathrm{C}$ state at $E_{x}=9.45 \mathrm{MeV}$ obviously falls in the first category, 
as expected from the reaction mechanism. Further work and experiments are needed to establish the analogs and fully understand the existence of nuclear molecules in the $A=10$ nuclei.

To conclude, the ${ }^{10} \mathrm{~B}+{ }^{10} \mathrm{~B}$ reactions (at 50 and $72 \mathrm{MeV}$ ) were measured yielding new results on clustering at high excitations of several isotopes in mass region around $A=10$. These results are important for completing the spectroscopy of light nuclei and picture of different structures that appear in this mass region. Even today, after many decades of intensive research, a number of subjects related to light atomic nuclei still offer surprises and interesting new phenomena.

\section{References}

[1] Hafstad L. R. and Teller E., Phys. Rev. 54 (1938) 681.

[2] Ikeda K., Tagikawa N. and Horiuchi H., Prog. Theor. Phys. Extra Suppl. (1968) 464.

[3] Horiuchi H. and Ikeda K., Prog. Theor. Phys. 40 (1968) 277.

[4] von Oertzen W., Freer M. and Kanada-En'yo Y., Phys. Rep. 432 (2006) 43.

[5] Freer M., Rep. Prog. Phys. 70 (2007) 2149.

[6] Wiringa R. B. et al., Phys. Rev. C 62 (2000) 014001.

[7] von Oertzen W., Z. Phys. A 354 (1996) 37; 357 (1997) 355.

[8] Ito M., Itagaki N., Sakurai H. and Ikeda K., Phys. Rev. Lett. 100 (2008) 182502.

[9] Ito M. and Itagaki N., Phys. Rev. C 78, (2008) 011602(R).

[10] Itagaki N. et al., Phys. Rev. C 77 (2008) 067301.

[11] Kobayashi F. and Kanada-En'yo Y., Phys. Rev. C 86 (2012) 064303.

[12] Ebran J. -P., Khan E., Nikšić and Vretenar D., Phys. Rev. C 90 (2014) 054329 .

[13] Soić N. et al., Europhys. Lett. 34 (1996) 7.

[14] Milin M. et al., Nucl. Phys. A 753 (2005) 263. 
[15] Freer M. et al., Phys. Rev. Lett. 96 (2006) 042501.

[16] Rogachev G. et al., Prog Theor. Phys Suppl. 196 (2012) 184.

[17] Suzuki D. et al., Phys. Rev. C 87 (2013) 054301.

[18] Tengblad O., Bergmann U. C., Fraile L. M., Fynbo H. O. U. and Walsh S., Nucl. Instrum. Meth. Phys. Res. A 525 (2004) 458.

[19] Torresi D. et al., Nucl. Instrum. Meth. Phys. Res. A 713 (2013) 11.

[20] Grassi L. et al., Nucl. Instrum. Meth. Phys. Res. A 767 (2014) 99.

[21] Uroić M. et al., Eur. Phys. J. A 51 (2015) 93.

[22] Jelavić Malenica D., Ph.D. thesis, University of Zagreb, 2015.

[23] Tilley D. R. et al., Nucl. Phys. A 745 (2004) 155.

[24] Charity R. J. et al., Phys. Rev. C 80 (2009) 024306. 